\title{
VIBRATIONS OF A PENDULUM CONSISTING OF A BOB SUSPENDED FROM A WIRE: THE METHOD OF INTEGRAL EQUATIONS*
}

\author{
BY
}

HYUN J. AHN

Indiana State University

\begin{abstract}
The vibrations of a vertical pendulum consisting of a bob suspended from a wire are studied by the method of integral equations and the composite method, respectively. The composite method combines the minimum principles and the method of integral equations. This problem consists of the fourth-order differential equation and the boundary conditions dependent on the eigenvalue parameter. Lower bounds are established for the lowest natural frequencies by both methods. Numerical results are presented. Both theoretical and computational efficiencies are illustrated and the method of integral equations is stressed.
\end{abstract}

1. Introduction. The problem of determining lower bounds is considered for the lowest frequencies for the vibrations of a stiff wire pendulum consisting of a bob suspended from a wire by the method of integral equations and the composite method. Handelman and Keller [6] derived the fourth order partial differential equation governing the motion of a stiff wire pendulum, separated it under the assumption that the solution is harmonic time-dependent and reduced it to the time-independent eigenvalue problem in dimensionless form:

$$
\begin{gathered}
\eta^{2} u^{i v}-\left[\{\alpha(1-x)+1\} u^{\prime}\right]^{\prime}=\alpha v^{2} u, \quad 0<x<1, \\
u(0)=u^{\prime}(0)=u^{\prime \prime}(1)=0, \\
\eta^{2} u^{\prime \prime \prime}(1)=u^{\prime}(1)-v^{2} u(1),
\end{gathered}
$$

where $u$ is the dimensionless time-independent displacement of the pendulum, $\eta^{2}$ is the dimensionless stiffness, $\alpha$ is the mass ratio of the wire to the bob, and $v$ is the dimensionless frequency that corresponds to the eigenvalue of this system of equations. The mathematical problem is then to establish lower bounds for the smallest eigenvalues of the above problem. A special feature of this problem is the dependence of the boundary condition on the eigenvalue parameter.

It is the purpose of this paper to study the above eigenvalue problem by the method of integral equations. The above problem is converted to an equivalent Fredholm inte-

* Received July 2, 1979. Portions of this paper form a part of the doctoral dissertation [1]. The author is deeply conscious of his debt to his advisor, Dr. Bruce E. Goodwin. 
gral equation whose kernel is meromorphic in $\lambda$ and depends on one solution of the differential equation $L[\phi]=0$ in section 2, [1], where

$$
L[\phi]=\eta^{2} \phi^{\prime \prime}-\{\alpha(1-x)+1\} \phi, \quad 0<x<1 .
$$

It is shown in section 3 that the spectrum of the above problem consists of an unbounded sequence of positive eigenvalues without accumulation points in the finite complex $\lambda$-plane using a few theorems of R. Iglisch [8]. Lower bounds depending on the parameters $\alpha$ and $\eta$ are established for the smallest eigenvalues by the method of integral equations in section 4, [1]. Lower bounds depending on $\alpha$ and $\eta$ are also established by the composite method which combines the minimum principles $[2,3,5]$ and the method of integral equations in section 5. Numerical results of the two methods are obtained in section 6. Both theoretical and computational efficiencies for the method of integral equations are illustrated in this paper.

The limiting case of zero stiffness, $\eta=0$, is that of a perfectly flexible pendulum for which the problem has been previously solved. We assume in the sections 2,3 and 4 that the stiffness is not zero, i.e., $\eta \neq 0$. A value $\lambda$ for which the integral equation

$$
u(x)=\lambda \int_{0}^{1} K(x, y ; \lambda) u(y) d y
$$

has nontrivial solutions is called an eigenvalue of the kernel $K(x, y ; \lambda)[8]$.

2. The integral equation formulation. Let $\lambda=v^{2}$. Then the eigenvalue problem introduced in the section 1 is rewritten as

where

$$
\begin{aligned}
M[u] & =\lambda u, \quad 0<x<1, \\
u(0) & =u^{\prime}(0)=u^{\prime \prime}(1), \quad \eta^{2} u^{\prime \prime \prime}(1)=u^{\prime}(1)-\lambda u(1),
\end{aligned}
$$

$$
M[u]=\alpha^{-1}\left[\eta^{2} u^{i v}-\left[\{\alpha(1-x)+1\} u^{\prime}\right]^{\prime}\right] .
$$

The problem (1) is converted into an integral equation whose kernel is the Green's function for the fourth order differential operator $M$ and the boundary conditions above [1]. The dependence of the boundary condition of (1) on the eigenvalue parameter causes the Green's function to be meromorphic in the eigenvalue parameter $\lambda$. Hence the equivalent integral equation is a linear homogeneous Fredholm integral equation of the second kind with the kernel dependent on the eigenvalue parameter.

Integrating the equation of (1) and applying the boundary condition of (1) to the resulting equation leads to

$$
\eta^{2} u^{\prime \prime \prime}(x)-\{\alpha(1-x)+1\} u^{\prime}(x)=-\alpha \lambda \int_{x}^{1} u(y) d y-\lambda u(1) .
$$

Letting $v(x)=u^{\prime}(x)$ in the equation above the problem (1) reduces to the second order integro-differential problem:

where

$$
\begin{aligned}
& L[v]=-\alpha \lambda \int_{x}^{1} u(y) d y-\lambda u(1), \\
& v(0)=v^{\prime}(1)=0,
\end{aligned}
$$

$$
L[v]=\eta^{2} v^{\prime \prime}-\{\alpha(1-x)+1\} v .
$$


The equation $L[v]=0$ is a type of the Airy differential equation [10]. According to the Sturmian theory of ordinary differential equations [9, P. 227] there exists a solution of $L[v]=0$ which has no zeros on $[0,1]$. We assume that $v(x)$ is a positive solution on $[0,1]$, and then $w(x)=v(x) \int_{0}^{x}[v(t)]^{-2} d t$ is the second linearly independent solution obtained by the method of reduction of order. Using these two linearly independent solutions and the definition [9] of Green's function we obtain the Green's function $K(x, y)$ for the operator $L$ and the boundary condition of $(2)$ :

$$
K(x, y)=\eta^{-2} S(x, y)+\eta^{-2} N(x, y),
$$

where

$$
\begin{aligned}
S(x, y) & =\begin{array}{ll}
-v(y) w(x), & 0 \leq x \leq y \leq 1, \\
-v(x) w(y), & 0 \leq y \leq x \leq 1,
\end{array} \\
N(x, y) & =v(1) v^{\prime}(1)\left[1+v^{\prime}(1) w(1)\right]^{-1} w(x) w(y) .
\end{aligned}
$$

Then the integro-differential problem (2) is equivalent to the integral equation

$$
v(x)=-\alpha \lambda \int_{0}^{1} \int_{z}^{1} K(x, z) u(y) d y d z-\lambda u(1) \int_{0}^{1} K(x, z) d z
$$

which is rewritten, by interchanging the order of the double integral, as

$$
u^{\prime}(x)=\lambda \int_{0}^{1} A(x, y) u(y) d y-\lambda u(1) \int_{0}^{1} K(x, y) d y,
$$

where

$$
A(x, y)=-\alpha \int_{0}^{y} K(x, s) d s .
$$

Integrating the equation (3) and using the boundary condition $u(0)=0$ it follows that

$$
u(x)=\lambda \int_{0}^{x} \int_{0}^{1} A(z, y) u(y) d y d z-\lambda u(1) \int_{0}^{x} \int_{0}^{1} K(z, y) d y d z
$$

from which we obtain by substituting 1 for $x$

$$
u(1)=\lambda[\delta(\lambda)]^{-1} \int_{0}^{1} \int_{0}^{1} A(t, y) u(y) d y d t
$$

provided that

$$
\delta(\lambda)=1+\lambda \int_{0}^{1} \int_{0}^{1} K(t, s) d s d t \neq 0 .
$$

Substituting (5) into (4) and interchanging the order of the double integrals of (4) the integral equation equivalent to the problem (1) is obtained:

$$
u(x)=\lambda \int_{0}^{1} G(x, y ; \lambda) u(y) d y
$$


where

$$
\begin{aligned}
G(x, y ; \lambda) & =T(x, y)+f(\lambda) X(x) X(y), \\
T(x, y) & =-\alpha \int_{0}^{x} \int_{0}^{y} K(t, s) d s d t, \\
X(x) & =\int_{0}^{x} \int_{0}^{1} K(t, s) d s d t
\end{aligned}
$$

and

$$
f(\lambda)=\alpha \lambda[1+X(1) \lambda]^{-1}
$$

It has been shown that the kernel of the equation (6) is the Green's function for the fourth order differential operator $M$ and the boundary condition of (1). It consists of a nondegenerate kernel $T(x, y)$, a degenerate kernel $X(x) X(y)$ and a meromorphic function $f$ in $\lambda$. The function $T(x, y)$ and $X(x) X(y)$ are positive definite $L_{2}$-kernels on $[0,1]^{2}$. The Green's function $G(x, y ; \lambda)$ depends on one solution $v(x)$ of $L[v]=0$ in the sense that the second solution $w(x)$ is obtained from $v(x)$ by the method of reduction of order.

The classical Green's function $G(x, y ; \lambda)$ does not exist at the simple pole $\lambda_{0}=-[X(1)]^{-1}>0$. In this case there exists the generalized Green's function $H\left(x, y ; \lambda_{0}\right)$ $\left[7\right.$, P. 300] for the operator $M$ and the boundary condition $u(0)=u^{\prime}(0)=u^{\prime \prime}(1)=0$, $\eta^{2} u^{\prime \prime \prime}(1)=u^{\prime}(1)-\lambda_{0} u(1)$.

3. Existence of eigenvalues. We use the notion of the Green's function along with a few theorems of R. Iglisch [8] to show that the set of the eigenvalues of the problem (1) consists of an unbounded sequence of positive eigenvalues without accumulation points in the finite complex $\lambda$-plane.

Theorem 1. The spectrum of the problem (1) consists of an unbounded sequence of positive eigenvalues without accumulation points in the finite complex $\lambda$-plane.

Proof. Let $\lambda_{0}<k<\infty$, where $\lambda_{0}=-[X(1)]^{-1}>0$. Then $G(x, y ; k)$ is the Green's function for the operator $M$ and the boundary conditions:

$$
\begin{aligned}
M[u] & =\lambda u, \quad 0<x<1, \\
u(0) & =u^{\prime}(0)=u^{\prime \prime}(1)=0, \eta^{2} u^{\prime \prime \prime}(1)=u^{\prime}(1)-k u(1) .
\end{aligned}
$$

Multiplying the equation above by $u(x)$ and integrating the resulting expression from $x=0$ to $x=1$ leads to

$$
\lambda=\frac{\eta^{2} \int_{0}^{1}\left(u^{\prime \prime}\right)^{2} d x+\int_{0}^{1}\{\alpha(1-x)+1\}\left(u^{\prime}\right)^{2} d x+k[u(1)]^{2}}{\alpha \int_{0}^{1} u^{2} d x}>0,
$$

after integrating by parts and using the boundary conditions above.

The function $G(x, y ; \infty)$, defined by $\lim _{k \rightarrow \infty} G(x, y ; k)$, is the Green's function for the operator $M$ and the boundary conditions:

$$
\begin{aligned}
M[u] & =\lambda u, 0<x<1, \\
u(0) & =u^{\prime}(0)=u(1)=u^{\prime \prime}(1)=0,
\end{aligned}
$$


where the boundary condition is the limiting case of that in (7) as $k \rightarrow \infty$. In a similar procedure applied to (7) it follows from (9) that

$$
\lambda=\frac{\eta^{2} \int_{0}^{1}\left(u^{\prime \prime}\right)^{2} d x+\int_{0}^{1}\{\alpha(1-x)+1\}\left(u^{\prime}\right)^{2} d x}{\alpha \int_{0}^{1} u^{2} d x}>0 .
$$

Since the function $G(x, y ; k)$ is real, continuous and symmetric in $x$ and $y$ on $[0,1]^{2}$ and is continuous in $k$ on $\left(\lambda_{0}, \infty\right)$, it follows from (8) and (10) that the spectrum of the kernel $G(x, y ; k)$ consists of an unbounded sequence of positive eigenvalues without accumulation points in $(-\infty, \infty)$ for each $k>\lambda_{0},[14$, p. 84]. Remembering that the function $f$ is meromorphic in $\lambda$ and letting $a=\alpha, b=0, c=X(1)$ and $d=1$ leads to $a c-b d=\alpha>0$. Therefore, the hypotheses of Theorems X-XII in [8] are satisfied. Furthermore, applying a similar procedure used for (7) to the problem (1) leads to

$$
\lambda=\frac{\eta^{2} \int_{0}^{1}\left(u^{\prime \prime}\right)^{2} d x+\int_{0}^{1}\{\alpha(1-x)+1\}\left(u^{\prime}\right)^{2} d x}{\alpha \int_{0}^{1} u^{2} d x+[u(1)]^{2}}>0 .
$$

This completes the proof.

4. Lower bounds by the method of integral equations. Lower bounds are established for the lowest eigenvalues using the integral equation (6) and Goodwin's method [4, Pp. 77-78].

Rewrite the equation (6) in the form

$$
u(x)-\lambda \int_{0}^{1} T(x, y) u(y) d y=\lambda f(\lambda) \int_{0}^{1} X(x) X(y) u(y) d y,
$$

and consider it temporarily as a nonhomogeneous Fredholm integral equation with the kernel $T(x, y)$. Let $R(x, y ; \lambda)$ be the resolvent kernel of the kernel $T(x, y)$, [8]. Then the equation (12) is converted to a homogeneous Fredholm integral equation with a degenerate kernel from which the Fredholm eigenvalue equation for (12) is obtained [4, Pp. 77-78]:

$$
1-\lambda f(\lambda) \int_{0}^{1} X(x)\left[X(x)-\int_{0}^{1} R(x, z ; \lambda) X(z) d z\right] d x=0 .
$$

Rearranging the equation (13) in terms of power series in $\lambda$ leads to the transcendental equation

$$
1+a_{1} \lambda+a_{2} \lambda^{2}+\cdots=0
$$

where

$$
\begin{aligned}
a_{1}= & X(1)-\int_{0}^{1} T(x, x) d x \\
a_{2}= & \left(\frac{1}{2} \int_{0}^{1} T(x, x) d x\right)^{2}-\frac{1}{2} \int_{0}^{1} T_{2}(x, x) d x \\
& -X(1) \int_{0}^{1} T(x, x) d x-\alpha \int_{0}^{1}[X(x)]^{2} d x .
\end{aligned}
$$


Let $\left\{\lambda_{n}: \lambda_{n}=v_{n}^{2}, \lambda_{i} \leq \lambda_{j}\right.$ if $\left.i \leq j, n=1,2, \ldots\right\}$ be the set of the eigenvalues of the problem (1). Then it follows from the Spiegel's formulae [13] that

$$
\begin{aligned}
\sum_{n=1}^{\infty} \lambda_{n}^{-1} & =-a_{1}, \\
\sum_{n=1}^{\infty} \lambda_{n}^{-2} & =a_{1}^{2}-2 a_{2}, \\
& =[X(1)]^{2}+2 \alpha \int_{0}^{1}[X(x)]^{2} d x+\int_{0}^{1} T_{2}(x, x) d x .
\end{aligned}
$$

The kernel $K(x, y)$ of the integral

$$
X(1)=\int_{0}^{1} \int_{0}^{1} K(t, s) d s d t \text { and } T(x, y)=-\alpha \int_{0}^{x} \int_{0}^{y} K(t, s) d s d t
$$

are continuous on $[0,1]^{2}$. Therefore, the series

$$
\sum_{n=1}^{\infty} \lambda_{n}^{-1} \text { and } \sum_{n=1}^{\infty} \lambda_{n}^{-2}
$$

are convergent (see [11]). The two equations above lead to the lower bounds for the lowest eigenvalue of the problem (1):

$$
\begin{aligned}
& v_{1} \geq\left(-a_{1}\right)^{-1 / 2}, \\
& v_{1} \geq\left(a_{1}^{2}-2 a_{2}\right)^{-1 / 4},
\end{aligned}
$$

where $\left(-a_{1}\right)^{-1 / 2}$ and $\left(a_{1}^{2}-2 a_{2}\right)^{-1 / 4}$, denote the lower bounds for the lowest natural frequencies of the problem (1) by using the first and second iteration, respectively. In this paper, for simplicity, the numerical result for (14) is presented. However, the lower bound $(15)$ is a better approximation than (14).

5. Lower bounds by the composite method. An alternative approach is introduced to obtain lower bounds for the smallest eigenvalue of the problem (1) by the composite method, which combines the minimum principles $[2,3,5]$ and the method of integral equations introduced in section 4.

Consider the equation (11) in which the right-hand side is the Rayleigh quotient for the problem (1). Let $V$ be the class of admissible functions that consists of all functions $v(x)$ with continuous fourth derivatives, satisfying the boundary conditions $v(0)=$ $v^{\prime}(0)=0$. Let $V^{\prime}$ be the class of functions $v(x)$ that consists of all functions with continuous second derivative satisfying $v(0)=0$. Then the class $V^{\prime}$ contains the class $V$. Consequently, the minimum principles [2, 3, 5] together with the Southwell [12] technique and equation (11) lead to

$$
\lambda \geq \eta^{2} \min _{v \in V} \frac{\int_{0}^{1}\left(v^{\prime \prime}\right)^{2} d x}{\alpha \int_{0}^{1} v^{2} d x+[v(1)]^{2}}+\min _{v \in V^{\prime}} \frac{\int_{0}^{1}\{\alpha(1-x)+1\}\left(u^{\prime}\right)^{2} d x}{\alpha \int_{0}^{1} v^{2} d x+[v(1)]^{2}}
$$

Let

$$
\beta \geq \min _{v \in V} \frac{\int_{0}^{1}\left(v^{\prime \prime}\right)^{2} d x}{\alpha \int_{0}^{1} v^{2} d x+[v(1)]^{2}}
$$


Then $\beta$ is the eigenvalue of the fourth order eigenvalue problem

$$
\frac{1}{\alpha} u^{1 v}=\beta u, \quad 0<x<1,
$$

with the required boundary conditions

$$
u(0)=u^{\prime}(0)=0,
$$

and the natural boundary conditions

$$
u^{\prime \prime}(1)=u^{\prime \prime \prime}(1)+\beta u(1)=0 .
$$

The eigenvalue equation of the problem (17) with the boundary conditions above is

$$
\alpha^{2} \beta^{2}-\cot (\alpha \beta) \operatorname{coth}(\alpha \beta)=0
$$

and the set of the eigenvalues consists of the positive roots of (18). We assume $\beta_{1}$ to be the smallest eigenvalue of (18). Let

$$
\mu \geq \min _{v \in V^{\prime}} \frac{\int_{0}^{1}\{\alpha(1-x)+1\}\left(v^{\prime}\right)^{2} d x}{\alpha \int_{0}^{1} v^{2} d x+[v(1)]^{2}} .
$$

Then $\mu$ is the eigenvalue of the second-order eigenvalue problem

$$
\frac{-1}{\alpha}\left[\{\alpha(1-x)+1\} u^{\prime}\right]^{\prime}=\mu u, \quad 0<x<1,
$$

with the required boundary condition $u(0)=0$ and the natural boundary condition $u^{\prime}(1)=\mu u(1)$. This system of equations is equivalent to the linear homogeneous Fredholm integral equation of the second kind whose kernel is the Green's function for the system

$$
g(x, y ; \mu)=k(x, y)+h(\mu) Y(x) Y(y)
$$

where

$$
\begin{aligned}
Y(x) & =\log \frac{\alpha(1-x)+1}{1+\alpha} \\
k(x, y) & =\left\{\begin{array}{lr}
Y(x), & 0 \leq x \leq y \leq 1, \\
Y(y), & 0 \leq y \leq x \leq 1,
\end{array}\right.
\end{aligned}
$$

and

$$
h(\mu)=\frac{\alpha \mu}{-\mu \log (1+\alpha)+\alpha}
$$

Applying the method of integral equation intróduced in section 4 to the kernel $g(x, y)$ leads to

$$
\mu_{1} \geq \sqrt{2} \alpha\left[\alpha^{2}+2 \alpha-2 \log (1+\alpha)\right]^{-1 / 2},
$$

where $\mu_{1}$ is obtained from the second iteration and assumed to be the smallest eigenvalue of the problem (20). 
Therefore, it follows from (16), (18) and (21) that the lower bounds for the lowest eigenvalue of the problem (1) by the composite method are given by

$$
v_{1} \geq\left[\eta^{2} \beta_{1}+\sqrt{2} \alpha\left\{\alpha^{2}+2 \alpha-2 \log (1+\alpha)\right\}^{-1 / 2}\right]^{1 / 2} .
$$

6. Numerical results. In this section numerical results are obtained to illustrate the efficiency, practicality, and accuracy of the method of integral equations. Since the lower bound (14) depends on one solution of the equation $L[u]=0$, an infinite series solution is employed. In actual computation $v(x)=\sum_{n=0}^{25} c_{n} x^{n}, c_{0}=1$, is used as an approximate solution to the infinite series solution $u(x)=\sum_{n=0}^{\infty} c_{n} x^{n}, c_{0}=1$. The basic idea of computing the lower bound (14) is to evaluate the multiple integrals involved recursively by Simpson's and the trapezoidal rules using a step size 0.02 and the approximate finite series solution. A FORTRAN program for computing the lower bound (14) has been written based on this idea and executed by IBM 360. The results are given in Figure 1.

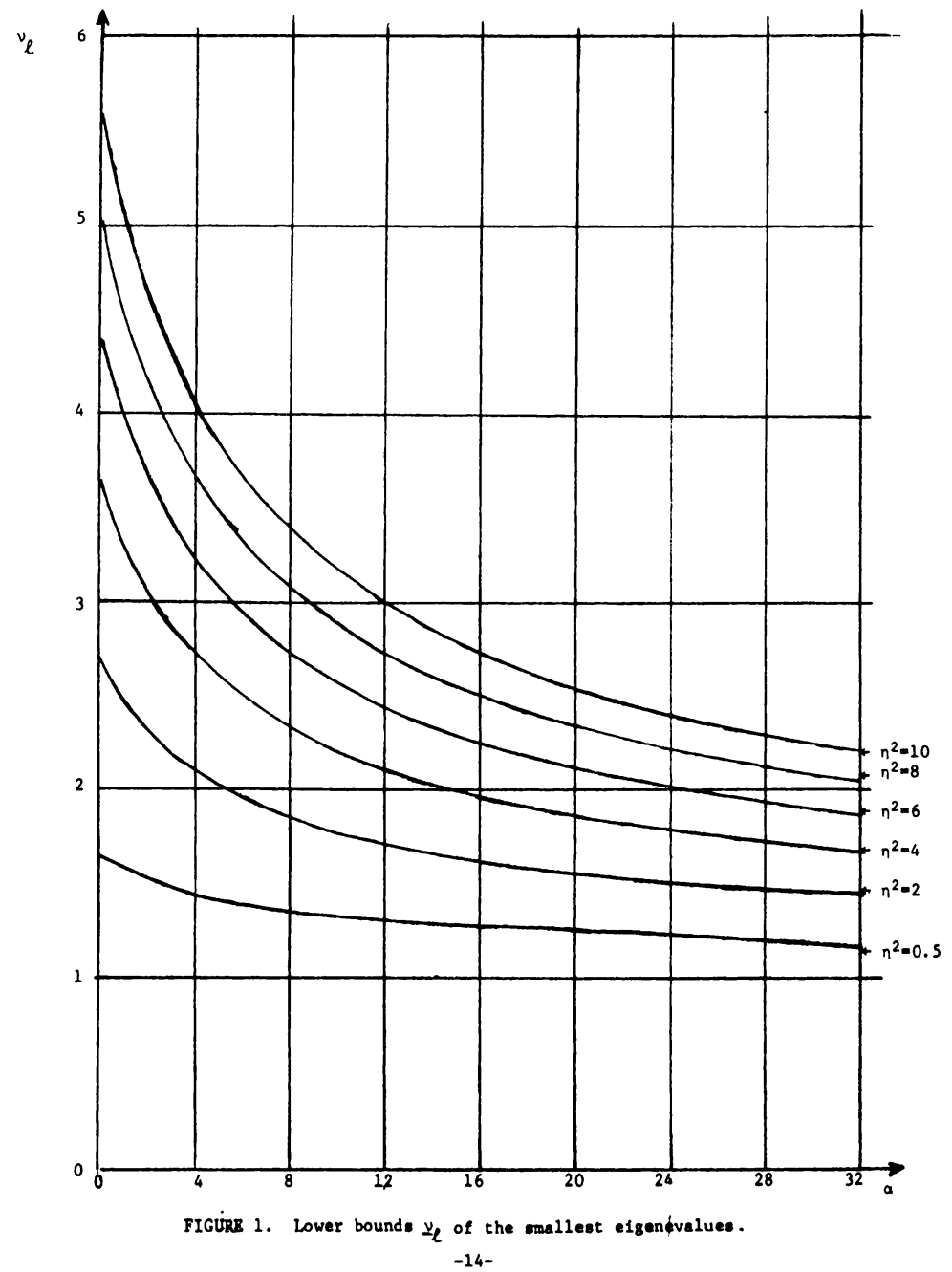

FIG. 1. Lower bounds $v_{l}$ of the smallest eigenvalues. 
The numerical results of the lower bound (22) by the composite method are obtained from elementary computations using Newton's method. The results are so close to the ones of (14) that they are hardly distinguishable for the scale of the dimensions chosen in Figure 1.

7. Conclusion. The Green's function for the fourth boundary value problem (1) has been analytically obtained. The efficiency and the practical importance of Green's functions were illustrated. There are characteristic difficulties in this problem since the boundary condition depends on the eigenvalue parameter. However, the reformulation of the problem (1) by an integral equation with the kernel, Green's function (6), dependent on the eigenvalue parameter enabled us to overcome the characteristic difficulties along with the remarkable results of $\mathbf{R}$. Iglisch and Goodwin's method.

Finally, we have shown how the method of integral equations and the composite method can be utilized to obtain the lower bounds of the lowest natural frequencies of the transverse vibrations of a pendulum consisting of a bob suspended from a wire. Without using a computer it might have been impossible to present the numerical results by the method of integral equations in this paper. A related result by the singular perturbation method can be found in [6].

\section{REFERENCES}

[1] H. J. Ahn, Operator-valued functions of the eigenvalue, Doctoral dissertation, University of Delaware, June, 1975

[2] W. E. Boyce, R. C. DiPrima, and G. H. Handelman, Vibrations of rotating beams of constant section, Proc. Second U.S. Natl. Congress of Appl. Mech. 122-152 (1954)

[3] R. Courant and D. Hilbert, Methods of mathematical physics, vol. 1, Interscience, New York, 1953

[4] B. E. Goodwin, On the realization of the eigenvalues of integral equations whose kernels are entire or meromorphic in the eigenvalue parameter, J. SIAM Appl. Math. 14, 65-85 (1966)

[5] G. H. Handelman, W. E. Boyce, and H. Cohen, Vibrations of a uniform, rotating beam with tip mass, Proc. Third U.S. Natl. Congress of Appl. Mech. 175-180 (1958)

[6] G. H. Handelman and J. B. Keller, Small vibrations of a slightly stiff pendulum, Proc. Fourth U.S. Natl. Congress of Appl. Mech., 195-202 (1962)

[7] F. B. Hildebrand, Method of applied mathematics, Prentice-Hall New Jersey, 1961

[8] R. Iglisch, Uber lineare Integralgleichungen mit vom Parameter abhangigem Kern, Math. Ann., 117, 129-139 (1939)

[9] E. L. Ince, Ordinary differential equations, Dover, New York, 1956

[10] H. Jeffreys and B. Jeffreys, Methods of mathematical physics, Cambridge University Press, New York, 1972

[11] A. V. Laginestra and W. E. Boyce, Convergence and evaluation of sums of reciprocal powers of eigenvalues of boundary value problems nonlinear in the eigenvalue parameter, SIAM J. Math. Anal. 5, 64-90 (1974)

[12] R. V. Southwell, Aero. Res. Comm. (U.K.), Reports and memoranda 486; H. Lamb and R. V. Southwell, The vibrations of a spinning disc, Proc. Roy. Soc. (A), 99, 272-280 (1921)

[13] M. R. Spiegel, The summation of series involving roots of transcendental equations and related applications, $J$. Appl. Phys. 24, 1103-1106 (1953)

[14] K. Yosida, Lectures on differential and integral equations, Interscience, New York, 1960 